\title{
Chair of WineHealth 2013 scientific advisory committee
}

\author{
Creina S. Stockley* \\ The Australian Wine Research Institute, Adelaide, SA, Australia
}

This collection of 16 papers was presented at the WineHealth 2013 International Wine and Health Conference. This conference was the seventh in the series of WineHealth International Wine and Health conferences and was held in Sydney, Australia on 18-20 July 2013. There were eight sessions in total comprising 28 presentations. These presentations considered the evolving epidemiological evidence on the relationship between alcohol and wine consumption and human health, in addition to recent scientific studies on the effects of wine consumption on different diseases of ageing and the influence of diet and lifestyle. Recent research on the biological effects and mechanisms of grape and wine components was also considered.

As was discussed in the opening Serge Renaud Memorial Lecture (Ellison 2014, pp. 81-84), and over the following days of the conference, epidemiological studies conducted since the publication of the 'French paradox' [1] have generally concluded that there is an inverse relationship between light to moderate consumption of wine and risk of cardiovascular disease. This inverse relationship can be extended to all-cause mortality, that is, regular light to moderate consumption reduces the risk of death from all causes compared to heavy consumption and lifelong abstinence, including death from cardiovascular and cerebrovascular diseases, dementia, diabetes and certain cancers [2-9]. These studies have been subsequently supported by in vitro, animal and ex vivo studies, as well as limited

${ }^{*}$ Corresponding author: Creina S. Stockley, AWRI, PO Box 197, Glen Osmond, SA 5064, Australia. E-mail: Creina.Stockley@awri. com.au. human in vivo studies, which have elucidated multiple biological mechanisms.

This emerging science is often not considered, however, when public health policy on alcohol is prepared, as policy generally focuses on the risk of short and long term harms to human health as consumption increases above moderation.

The WHO's Projected global death for selected causes, 2005 to 2030, suggests that death from ischaemic heart disease, cerebrovascular disease and cancers, in particular, will continue to increase [10]. Cardiovascular diseases accounted for $34 \%$ of the total number of deaths in Australia in 2008, and 18\% of the overall burden of disease, with coronary heart disease and stroke contributing over $80 \%$ of this burden [11]. Alcohol consumption per se, however, accounted for $-4.7 \%$ of the total cardiovascular disease burden. Australia shares similar demographics and the same 19 leading causes of burden as other high income developed countries [12].

Globally, the population aged 60 years and over is projected to nearly triple by 2050 , while the population aged 80 years and over is projected to experience a more than five-fold increase. In Australia, between now and 2050 the number of older individuals (65 to 84 years) is expected to more than double; and the number of very old individuals ( 85 and over) is expected to more than quadruple from 0.4 million people today to 1.8 million in 2050 (www.treasury.gov.au/igr) igr2010/Overview/html/overview). Increased numbers of older individuals may have implications for expenditure on income support, housing and health services, 
although a healthy, independent older population can also form a valued social resource, for example in providing care for others, sharing skills and knowledge and engaging in volunteer activities. Consequently, simple dietary measures such as light to moderate alcohol and wine consumption to supplement a healthy exercise and nutrition routine, or as an adjunct to prescription medicines when appropriate, may be needed to maintain an ageing population.

Indeed, Simons (2014, pp. 85-90) showed in the 20-year, longitudinal study of 2,805 Australians aged 60 years and over (which examined the relationship between alcohol consumption and mortality) that the inclusion of any alcohol in the diet increased the lifespan of both men and women by 12 months. All subjects in the light ${ }^{1}$ and moderate ${ }^{2}$ consumption categories had a significant $25 \%$ lower risk of all cause mortality compared with heavier consumption. The risk was $20 \%$ and $28 \%$, respectively, when compared with abstinence. Importantly, there appeared to be significant protection against cardiovascular disease at low alcohol consumption and this relationship did not appear to be impacted or mediated by diabetes, hypertension, obesity or HDL cholesterol, which are independent risk factors for cardiovascular disease. Similar protection against risk factors for cardiovascular disease was observed for 2,900 US women approaching 60 years of age who consumed light to moderate amounts of wine in the 8-year longitudinal Study of Women's Health Across the Nation (Janssen et al. 2014, pp. 91-99).

The Dubbo Study of Australian Elderly also showed a significantly lower risk of onset of dementia with light to moderate alcohol consumption. Complementary to these observations, in the study by Scholey et al. (2014, pp. 133-138) light to moderate consumption of red wine containing an additional $100 \mathrm{mg}$ of the wine-derived phenolic compound, resveratrol, was also observed to improve cognitive functioning in 16 elderly individuals. The improvement was in demanding cognitive processing, while red wine alone was superior in terms of performing performance of an attentional task. This suggests that the alcohol and resveratrol components of red wine may have different effects on brain function; red wine is a significant dietary source of resveratrol. A rat animal model

\footnotetext{
${ }^{1}$ Light consumption was 1-14 drinks/week for men and 1-7 drinks/week for women.

${ }^{2}$ Moderate consumption was $15-24$ drinks/week for men and 8-14 drinks/week for women.
}

administered Champagne wine also showed improvements in spatial working memory, a function which is impaired in individuals with dementia, via modulation of brain signalling (Corona et al. 2014, pp. 125-132).

A common message in the concluding comments of many of the papers presented was that light to moderate wine consumption should not replace a healthy diet and lifestyle but should be an adjunct to it to promote healthy ageing, and that this information should be imparted by medical practitioners to their patients. This science is still in its infancy, however, and many more clinical and epidemiological studies are required to fully know and better understand the effects of wine and its core components on human health. Future potential research questions to ponder in order to better inform public health policy, therefore, include the following:

- Large or multicentre, randomised controlled clinical studies to assess whether moderate wine consumption truly has protective effects on human health.

- What dose of wine provides protective effects?

- How significant are phenolic components compared to the alcohol component of wine?

- Are there positive and negative interactions between the alcohol and phenolic compound components?

- What is the minimum concentration of phenolic compounds necessary to achieve a biological/ clinical effect and is the dose-response relationship thereafter linear or exponential?

- Are all the classes of phenolic compounds equally active or biologically/clinically effective?

- Are the necessary concentrations of phenolic compounds generally observed in grapes and wine or are they realistically achievable in grapes and wine by manipulating vineyard and oenological practices?

- What are the main pharmacological and physiological differences between grape- and winederived phenolic compounds?

- How often does a wine-derived phenolic compound need to be consumed for the occurrence of short-, medium- and long-term protective effects, as well as a continuation of these effects?

- What is the impact of a diet that includes wine compared to a diet including other significant sources of phenolic compounds as well as exercise on human health and longevity? 


\section{References}

[1] Renaud S, de Lorgeril M. Wine, alcohol, platelets, and the French paradox for coronary heart disease. Lancet. 1992;339: 1523-6.

[2] Grønbæk M, Becker U, Johansen D, Gottschau A, Schnohr P, Hein HO, Jensen G, Sorensen TIA. Type of alcohol consumed and mortality from all causes, coronary heart disease, and cancer. Ann Intern Med. 2000;133:411-9.

[3] Zuccalà G, Onder G, Pedone C, Cesari M, Landi F, Bernabei $\mathrm{R}$ and Cocchi A, Gruppo Italiano di Farmacoepidemiologia nell'Anziano Investigators Dose-related impact of alcohol consumption on cognitive function in advanced age: Results of a multicenter survey. Alcohol Clin Exp Res. 2001;25: 1743-8.

[4] Briggs NC, Levine RS, Bobo LD, Haliburton WP, Brann EA, Hennekens $\mathrm{CH}$. Wine drinking and risk of non-Hodgkin's lymphoma among men in the United States: A population-based case-control study. Am J Epidemiol. 2002;156(5):454-62.

[5] Wannamethee SG, Shaper AG, Perry IJ, Alberti KG. Alcohol consumption and the incidence of type II diabetes. J Epidemiol Community Health. 2002;56:542-8.
[6] Wannamethee SG, Camargo CA Jr, Manson JE, Willett WC, Rimm EB. Alcohol drinking patterns and risk of type 2 Diabetes Mellitus among younger women. Arch Intern Med. 2003;163:1329-36.

[7] Pedersen A, Johansen C, Grønbaek M, Relations between amount and type of alcohol and colon and rectal cancer in a Danish population based cohort study. Gut. 2003;52:861-7.

[8] McDougall GJ Jr, Becker H, Areheart KL, Older males, cognitive function, and alcohol consumption. Issues Mental Health Nursing. 2006;27:33-53.

[9] Bantle AE, Thomas W, Bantle JP. Metabolic effects of alcohol in the form of wine in persons with type 2 diabetes mellitus. Metabolism. 2008;57(2):241-5.

[10] World Health Organisation World Health Statistics 2007. World Health Organisation, Geneva, Switzerland (2007) http://www.who.int/whosis/whostat2007_10highlights.pdf

[11] Australian Institute of Health and Welfare Cardiovascular disease: Australian facts, 2011. Cat. no. CVD 53; Canberra; 2011.

[12] Lopez AD, Mathers CD, Ezzati M, Jamison DT and Murray CJL, Global burden of disease and risk factors. A copublication of Oxford University Press and The World Bank; 2006. http://files.dep2.org/pdf/GBD/GBD.pdf 